\title{
Numerical Study of Single Iron(III) Nitrate Nonahydrate/Ethanol Droplet Evaporation in Humid Air
}

\author{
Praveen Narasu ${ }^{1}$, Alexander Keller ${ }^{2}$, Maximilian Kohns ${ }^{2}$, Hans Hasse ${ }^{2}$, Eva Gutheil ${ }^{1}$ \\ ${ }^{1}$ Interdisziplinäres Zentrum für Wissenschaftliches Rechnen, Universität Heidelberg, Germany \\ Im Neuenheimer Feld 205, 69120 Heidelberg, Germany \\ praveen.narasu@uni-heidelberg.de; gutheil@iwr.uni-heidelberg.de \\ ${ }^{2}$ Lehrstuhl für Thermodynamik, Technische Universität Kaiserslautern, Germany \\ Erwin-Schrödinger-Straße 44, 67663 Kaiserslautern, Germany \\ alexander.keller@mv.uni-kl.de, maximilian.kohns@mv.uni-kl.de, hans.hasse@mv.uni-kl.de
}

\begin{abstract}
The heating and evaporation of spherically symmetric droplets consisting of a precursor solution of iron(III) nitrate nonahydrate (INN) and ethanol for nanoparticle synthesis in spray flames is studied numerically. The multicomponent droplets are at standard conditions and the evaporation is initiated through the elevated temperature of the convective ambient air. The liquid mixture properties of INN and ethanol used in the model are fits to new experimental data which are valid in the temperature range of $293.15 \mathrm{~K}$ through $333.15 \mathrm{~K}$ and for mass fractions of INN in ethanol up to 0.34. In general, there are two different pathways through which the nanoparticle synthesis may occur from the precursor solution. In the first pathway, the particle forms directly inside the liquid precursor solution, and in the second pathway, the droplet is transferred entirely into the gas phase from which the nanoparticle may form. The INN/ethanol precursor droplet is treated as a three-component solution consisting of ethanol, iron(III) nitrate, and water, where the INN consists of the latter two. The process is initially governed by the ethanol evaporation and later by water evaporation since ethanol is more volatile component. Further decomposition and liquid-phase reactions may occur inside the precursor droplet, eventually leaving over an iron (III) nitrate particle or transferring the iron (III) nitrate into the gas phase, which happens beyond the thermal decomposition temperature of $403 \mathrm{~K}$ beyond which the iron (III) nitrate may decompose into gaseous $\mathrm{Fe}_{2} \mathrm{O} 3$, so that the entire INN/ethanol droplet transforms into the gas phase. The study presents simulations of the evaporation of the INN/ethanol droplet for both situations.
\end{abstract}

Keywords: multicomponent droplet evaporation, thermal decomposition, precursor solution, iron(III) nitrate nonahydrate, ethanol, numerical study

\section{Introduction}

Spray flame synthesis of functional nanoparticles is a very promising method to produce nanoparticles with special characteristics. While the synthesis of nanoparticles in gas flames is well established, for instance for the production of fumed silica [1] and carbon black [2], flame spray pyrolysis (FSP) has been studied to a lesser extent. FSP is characterized through processes including the heating, evaporation, and transformation of the precursor solution into the gas phase and the combustion of the resulting system as well as the formation of the nanoparticles. The synthesis of nanoparticles in FSP is of particular interest in technical processes where the focus lies on special properties of the nanoparticles which include the purity of the final product and the flexibility of the experimental operations [3]. Teoh et al. [4] study these processes in various application areas including sensors, functional pigments, fuel cells, bioelectronics, solar cells, and batteries. Therefore, it is necessary to understand how to control both the combustion characteristics of the flame and the fluid dynamics in order to achieve the before-mentioned challenges. There is a vast of publications that concern different materials. Rittler et al. [5] performed large eddy simulations of the production of silica nanoparticles from flame spray pyrolysis using ethanol/hexamethyldisiloxane (HMDSO) mixtures as precursor and oxygen as dispersion gas. This study focuses on the understanding of the interaction between spray evaporation, gas phase combustion, and synthesis of nanoparticles. The droplet evaporation has been incorporated using the distillation-limit model. Heine and Pratsinis [6] studied zirconium dioxide $\left(\mathrm{ZrO}_{2}\right)$ particle formation by FSP theoretically and compared with experimental data of the particle size distribution. The interaction between droplet evaporation, combustion, particle formation and growth has been studied for the synthesis 
of $\mathrm{ZrO}_{2}$ particles by using a detailed droplet evaporation model based on the work of Abramzon and Sirignano [7], which was extended for multicomponent droplet evaporation [8] and combined with a sectional population balance model that accounts for nucleation, coagulation, and sintering. Neto et al. [3] also modeled the production of $\mathrm{ZrO}_{2}$ nanoparticles via FSP and the combustion of the precursor-solvent mixture is described through seven sets of different chemical reaction mechanisms to analyze their influence on the flame temperature and the particle evolution within the process. The reacting turbulent multiphase flow was described by a Eulerian-Lagrangian approach, and $\mathrm{ZrO}_{2}$ nanoparticle growth has been estimated by solving the population balance equations from a mono-disperse model based on coagulation and sintering. The models were able to predict the temperatures and primary particle diameters within 9 $\%$ and $6 \%$ accuracies of experimental values, respectively.

FSP is typically dominated by the liquid phase processes of the spray. For the precursor solutions, a major difficulty is the typically unknown physical properties of the precursor and the multicomponent droplet evaporation process that must be incorporated into the global model. The present study concerns the modeling and simulation of the heating, evaporation, and thermal decomposition of INN/ethanol droplets in convective air where the focus lies on the investigation of the conditions under which particles are directly formed inside the precursor droplet or where the precursor droplet is completely transferred into gas phase from which nanoparticle formation may occur.

\section{Mathematical Model}

The model concerns the convective heating, evaporation, and thermal decomposition of a single INN/ethanol droplet. In principle, there are two different pathways for the evaporation of a precursor droplet which are schematically displayed in Fig. 1. At low instantaneous droplet temperatures $T_{\mathrm{d}}$ below the thermal decomposition temperature, $T_{\text {th }}$, the droplet heats up and the solvent evaporates, leaving the solute in the particle phase behind as shown in the left part of Fig. 1. $T_{\mathrm{d}, 0}$ denotes the initial precursor droplet temperature. If the instantaneous droplet temperature is equal or above $T_{\text {th }}$, thermal decomposition occurs and the precursor droplet is completely transferred into the gas phase. The latter pathway is preferred in FSP even though the first may occur in colder regions of the flow for instance, just after liquid injection. The present model is suitable to capture both pathways. A spherically symmetric droplet is assumed at atmospheric pressure in a convective gas stream at low droplet Reynolds numbers up to about twenty. This assures that no inner recirculation of the droplet and no flow separation in the wake of the droplet occur. For the liquid system of INN/ethanol at atmospheric pressure, the distillation-limit model for the liquid temperature is used to describe the droplet heating. Thus, both uniform droplet temperature and species concentrations inside the droplet are assumed. In the present model, the droplets are assumed spherical at all times, the solubility of air in the liquid is negligible, mass diffusion due to temperature and pressure gradients is negligible, the gas phase is in a quasi-steady state, the droplet evaporates in an inert environment without chemical reactions and the heat transfer due to radiation is negligible [8].

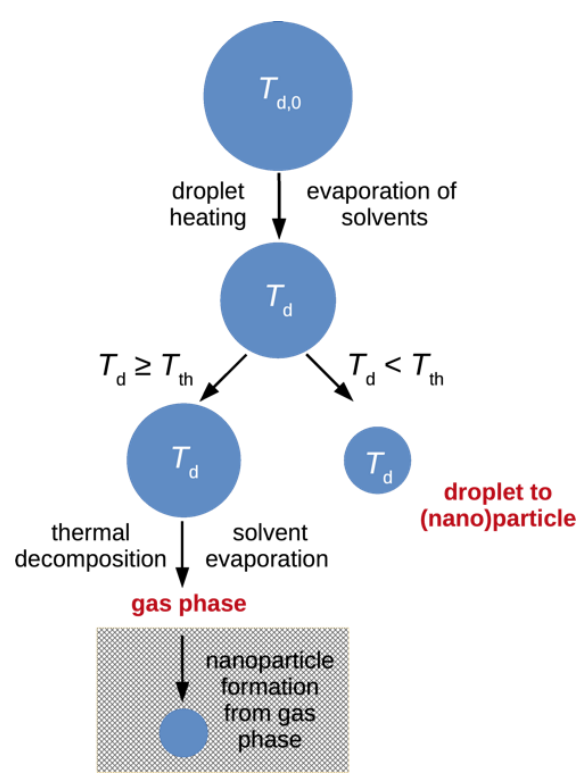

Fig. 1: Schematic presentation of the pathways for the heating, evap-oration, and possible thermal de-composition of a precursor droplet.

\subsection{Thermal Decomposition}

The experimental conditions are chosen following the experiment performed by Schneider et al. [9]. The initial mass fraction of the INN in ethanol varies between 0.0125 and 0.025 . The liquid mixture properties of INN and ethanol used in the model are fits to new experimental data for the liquid properties of INN in ethanol which are valid within the temperature range of $293.15 \mathrm{~K}$ through $333.15 \mathrm{~K}$ and for mass fractions of INN in ethanol up to 0.34. For ambient gas temperatures below the thermal decomposition temperature of $403 \mathrm{~K}$ for the INN, the solvent evaporates and leaves the 
iron(III) nitrate particle behind. If the thermal decomposition temperature is exceeded, the iron(III) nitrate may thermally decompose into iron(III) oxide, $\mathrm{Fe}_{2} \mathrm{O}_{3}$, and the entire liquid droplet transforms into the gas phase, cf. Fig. 1. The gaseous mixture burns in a flame in which the nanoparticle then may be generated. In the model for the INN/ethanol droplet heating, heating, evaporation, and thermal decomposition, the INN is treated as the two components iron(III) nitrate and water. Therefore, the precursor droplet consists of the three components iron(III) nitrate, water, and ethanol. The steps in converting the INN into the gas phase include the global reactions

and

$$
\mathrm{Fe}\left(\mathrm{NO}_{3}\right)_{3} .9 \mathrm{H}_{2} \mathrm{O} \rightarrow \mathrm{Fe}\left(\mathrm{NO}_{3}\right)_{3}+9 \mathrm{H}_{2} \mathrm{O}_{(\mathrm{l})}
$$

$$
\mathrm{H}_{2} \mathrm{O}_{(\mathrm{l})} \rightarrow \mathrm{H}_{2} \mathrm{O}_{(\mathrm{v})}
$$

Moreover, the evaporation of the solvent ethanol must be considered. Gadalla and Yu [10] report that the thermal decomposition of INN in air takes place via complex reaction steps, however, they do not give any kinetic reaction rates for these reactions. $\mathrm{Mu}$ and Perlmutter [11] provide the reaction kinetic data for the thermal decomposition of INN in an atmosphere of nitrogen, but they do not provide any reaction mechanism for the same. Mu and Perlmutter [11] observe that the INN starts decomposing into $\mathrm{Fe}\left(\mathrm{NO}_{3}\right)_{3}$ at $305 \mathrm{~K}$ and at $403 \mathrm{~K}, \mathrm{Fe}\left(\mathrm{NO}_{3}\right)_{3}$ starts to decompose into $\mathrm{Fe}_{2} \mathrm{O}_{3}$ for which the reaction kinetic data are provided. However, Mu and Perlmutter [11] do not propose an exact mechanism for the thermal decomposition of INN. Thus, the conclusions following Mu and Perlmutter [11] is that reaction

$$
2 \mathrm{Fe}\left(\mathrm{NO}_{3}\right)_{3} \rightarrow \mathrm{Fe}_{2} \mathrm{O}_{3}+3 \mathrm{~N}_{2} \mathrm{O}_{5}
$$

may be added to the reactions in Eqs. (1) and (2) to complete the global reaction of INN into the gas phase

$$
2\left[\mathrm{Fe}\left(\mathrm{NO}_{3}\right)_{3} .9 \mathrm{H}_{2} \mathrm{O}\right] \rightarrow \mathrm{Fe}_{2} \mathrm{O}_{3(\mathrm{v})}+6 \mathrm{NO}_{2(\mathrm{v})}+1.5 \mathrm{O}_{2(\mathrm{v})}+18 \mathrm{H}_{2} \mathrm{O}_{(\mathrm{v})}
$$

The present model for reactions Eqs. (1) and (2) corresponds to that of the evaporation of the ethanol and the water [14] leaving over the iron(III) nitrate, which decomposes following the reaction in Eq. (3) for which the reaction rate is determined in terms of $\alpha$, which is the normalized mass of the iron(III) nitrate $\alpha=\left(m_{0}-m_{\mathrm{t}}\right) /\left(m_{0}-m_{\mathrm{e}}\right)$ where $m_{0}$ is the initial mass of iron(III) nitrate, $m_{t}$ denotes the mass of iron(III) nitrate at time $t$ and $m_{\mathrm{e}}$ is the final mass of iron(III) nitrate which is assumed to be zero. The time derivative of $\alpha$ yields [13]

$$
\frac{\mathrm{d} \alpha}{\mathrm{d} t}=k_{0}(1-\alpha)^{n} \mathrm{e}^{-E /(\mathrm{R} T)}
$$

with the pre-exponential factor $k_{0}=2.6010^{44} \mathrm{~s}^{-1}$, the reaction order $n=2 / 3$, and the activation energy $E=376.98 \mathrm{~kJ} / \mathrm{mol}$. $\mathrm{R}$ is the universal gas constant, and $T$ denotes temperature. The thermal decomposition of the INN occurs for temperatures beyond $403 \mathrm{~K}$, transferring the precursor droplet entirely into the gas phase. For the completion of the model, the heating and evaporation of the water and the ethanol must be described [12].

\subsection{Convective Droplet Heating and Evaporation}

The droplet evaporation is described by the rapid-mixing model the applicability of which for the present conditions has been discussed by Narasu et al. [12] for the heating and evaporation of bi-component ethanol/water droplets in convective air. The study includes the Abramzon and Sirignano [7] model that was extended to account for the mass evaporation rate of a multicomponent droplet by Brenn et al. [8]. Narasu et al. [12] present a validation of the rapid-mixing and the distillationlimit models for the ethanol/water evaporation in convective air. 


\subsection{Physical Properties}

Keller et al. [13] measured the thermophysical properties of the solutions of INN in solvent mixtures of ethanol and water at atmospheric pressure where solvent compositions range from pure ethanol to pure water and INN molalities up $1.3 \mathrm{~mol} / \mathrm{kg}$ corresponding to a mass fraction of about 0.34 . The measured data include the density, viscosity, thermal conductivity, and isobaric specific heat capacity. The experimental relative standard uncertainty ranges from $0.2 \%$ for density to $10 \%$ for the mixture isobaric heat capacity. From the experimental thermophysical data, empirical correlations [13] for these properties were established. These thermophysical properties are valid within the temperature range from $288.15 \mathrm{~K}$ to $333.15 \mathrm{~K}$. The thermal decomposition temperature of the INN is $403 \mathrm{~K}$ [11] and the experimental properties are extrapolated. Variable liquid properties of the water and the ethanol as well as for the gas phase are taken from the study of Poling and Prausnitz [14]. The thermal conductivity of the gas mixture is calculated using the Wassiljewa equation. Wilke's rule is employed to evaluate the viscosity of the gas mixture in the film. Diffusivity of water vapor in air and that of ethanol vapor in air is evaluated using the Chapman and Enskog equation and the Fuller equation, respectively. The vapor pressure of both water and ethanol is calculated using the Antoine equation. The temperature dependence of the latent heat of evaporation is described using the extended Watson equation [15]. It is expected that the liquid mixture may not be ideal, and therefore, non-ideality is considered through the use of activity coefficients of ethanol and water through the UNIFAC method [16] described in detail by Narasu et al. [12]. The activity coefficient of the INN/ethanol mixture is not available and is neglected.

\section{Results and Discussion}

Focus is the two different pathways for the INN/ethanol droplet evaporation under combustion conditions relevant in spray flames synthesis of nanoparticles. The study concerns the initial droplet radii of $5 \mu \mathrm{m}$, for ambient air temperatures of $400 \mathrm{~K}$ and $1200 \mathrm{~K}$ and initial air velocities of 4 and $8 \mathrm{~m} / \mathrm{s}$. Two different initial INN mass fractions of 0.025 and 0.0125 in the precursor solution are considered. The ambience is either dry or humid air where relative humidity up to $10 \%$ is investigated. These conditions are chosen following the experimental conditions of a typical FSP burner [9]. For the present conditions, the Weber numbers stay well below the critical Weber number for which droplet breakup may occur and below thermal and mass Peclet numbers for which the distillation-limit model and the rapidmixing models are valid [12]. First, the ambient temperature of $400 \mathrm{~K}$ is studied for which the thermal decomposition does not occur because the temperature is below the thermal decomposition temperature of $403 \mathrm{~K}$, and then, higher ambient initial gas temperatures are considered.

\subsection{Droplet-to-Particle}

Figures 2 and 3 present the results of the parameter study for the evaporation of an INN/ethanol droplet at ambient temperature of $400 \mathrm{~K}$ which is below the thermal decomposition temperature so that after heating, the liquid ethanol and the water evaporate leaving the solid particle behind.

The left part of Fig. 2 shows the temporal change of the normalized droplet surface area for the different conditions under consideration and the right part of the figure presents the temporal evolution of mass evaporation rate of the components ethanol and water. For the droplet heating and evaporation in dry air, the process takes about $3.0 \mathrm{~ms}$ to 3.5 $\mathrm{ms}$ for the low and the enhanced ambient air velocity, respectively [12]. For the higher initial INN mass fraction, the final particle size is larger than for the smaller initial INN mass fraction as expected. The increase of relative humidity in air to $5 \%$ and $10 \%$ has a pronounced effect on the evaporation process. First, the preferential evaporation of ethanol is more pronounced in humid air compared to that in dry air because of the retardation of water evaporation due to the water condensation at the droplet surface seen in the right part of Fig. 2. The evaporation of water is reduced as can be seen from the reduced slope with increased relative humidity in its evaporation behavior following almost the wellknown $d^{2}$-law. However, the evaporation deviates from the $d^{2}$-law due to the variable properties of the liquids.

Figure 3 shows the temperature and the mass fractions of various species at the droplet surface for the lower ambient gas velocity in air with $0 \%$ (left) and $10 \%$ (right) relative humidity, respectively, in the convective air stream. For dry air, a very gradual increase of the droplet surface temperature during which the ethanol inside the droplet starts to evaporate is found - during this period of about $2 \mathrm{~ms}$, the water content inside the droplet remains almost constant after 

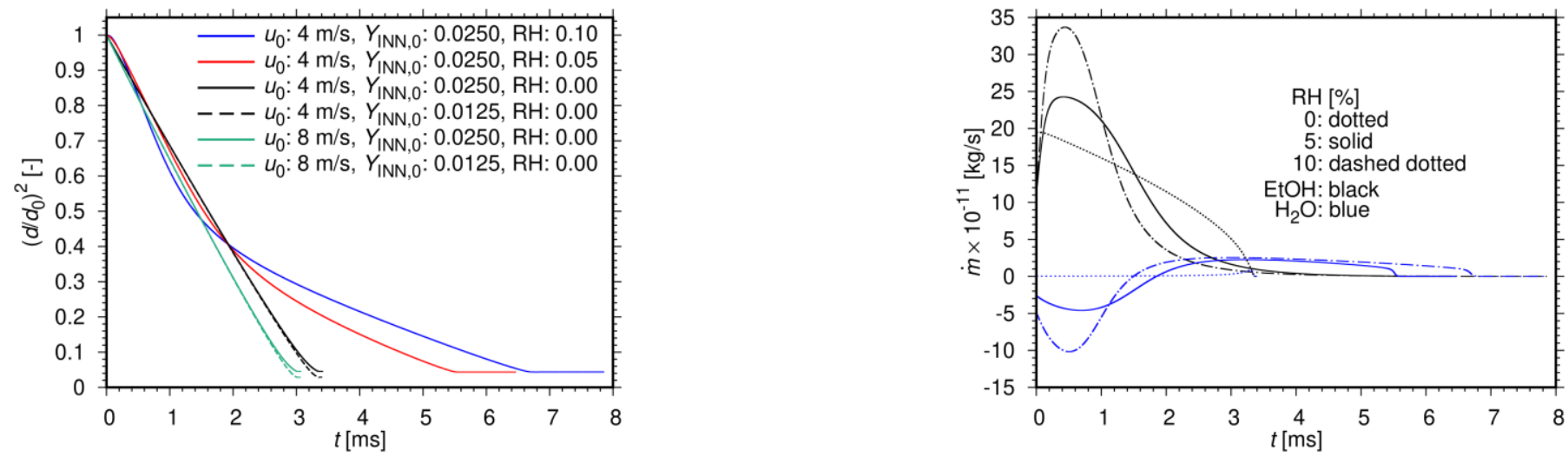

Fig. 2: Initial conditions: $r_{\mathrm{d}, 0}=5 \mu \mathrm{m}, T_{1,0}=293.15 \mathrm{~K}, p=1$ bar, $T_{\mathrm{g}, 0}=400 \mathrm{~K}$. Left: Normalized droplet surface area with time for various initial conditions. Right: Temporal evolution of mass evaporation rate of ethanol and water for $u_{0}=4 \mathrm{~m} / \mathrm{s}$ and $Y_{\mathrm{INN}, 0}=0.025$.
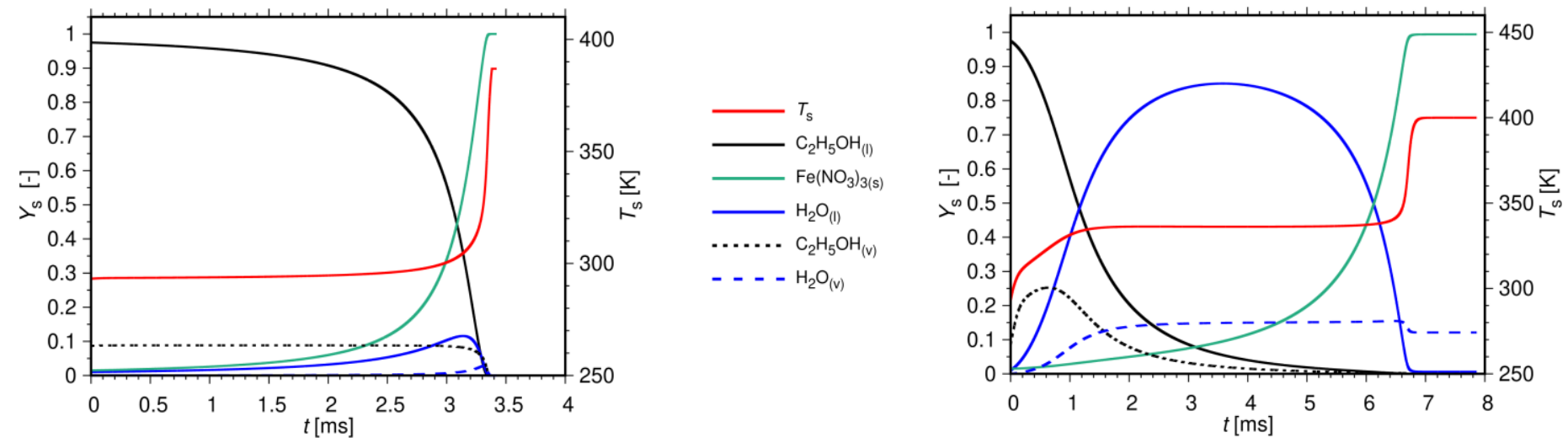

Fig. 3: Mass fractions of species and gas temperature at the droplet surface. Initial conditions: $r_{\mathrm{d}, 0}=5 \mu \mathrm{m}, T_{1,0}=293.15 \mathrm{~K}, p=1$ bar, $T_{\mathrm{g}, 0}=400 \mathrm{~K}, u_{0}=4 \mathrm{~m} / \mathrm{s}, Y_{\mathrm{EtOH}, 0}=0.975, Y_{\mathrm{H} 2 \mathrm{O}, 0}=0.011, Y_{\mathrm{IN}, 0}=0.014$. Left: $\mathrm{RH}=0 \%$. Right: $\mathrm{RH}=10 \%$.

which it increases due to the enhanced ethanol evaporation. Ethanol evaporation is preferred compared to that of water because of the higher volatility of ethanol compared to water. A major increase of droplet surface temperature occurs after about $2.8 \mathrm{~ms}$ which is the phase during which the ethanol completely evaporates and the water vapor content rises. Eventually, the solid iron(III) nitrate is left behind, terminating the process after about $3.4 \mathrm{~ms}$.

An increase of relative humidity from zero to $10 \%$ in the ambient air (right part of Fig. 3) strongly affects the evaporation characteristics. The most striking difference compared to the process in dry air is the initial increase of droplet surface temperature which increases with higher relative humidity. The level of the final surface temperature is also somewhat higher compared to the situation in dry air. This is an effect associated with the condensation of water at the droplet surface as seen from the temporal evolution of the mass evaporation rates of water and ethanol displayed in Fig. 2 (right part) for the two different values of relative humidity under consideration. During the condensation process, heat is released which increases the droplet surface temperature. A comparison of Figs. 2 and 3 (right parts) shows that the time during which condensation occurs, equals that of the increase of droplet surface temperature which is longer for the lower relative humidity of $5 \%$ compared to $10 \%$, but less pronounced. The final droplet surface temperatures are higher than the boiling temperatures of the liquids which is due to the humidity of the convective air. A closer look to the particle characteristics reveals that the total process time is pronouncedly prolonged for the higher relative humidity in the ambient air. The final particle size, however, is barely affected by the different relative humidity. Towards the end of droplet evaporation process, the mass fraction and the Spalding mass transfer number, $B_{\mathrm{M}}$, of both ethanol and the water decrease to zero. Thus, droplet evaporation terminates and the solid particle is left behind. 
The final particle radii that result from the droplet-to-particle simulation are between around $800 \mathrm{~nm}$ and 1,000 nm depending on the initial INN mass fraction of the precursor droplet. The targeted particle sizes are smaller and the aim produce particles from the gas phase so that next, the droplet evaporation and thermal decomposition will be discussed lead to transfer of the precursor droplet into gas phase.

\subsection{Droplet Evaporation and Thermal Decomposition}

In order for the precursor droplet to transform into gas phase, the ambient gas temperature is taken as $1200 \mathrm{~K}$ which lies above the thermal decomposition temperature.

Figure 4 shows the mass evaporation rate (left part) with time and the normalized droplet surface area (right part) with time for a pure ethanol droplet [12], a bi-component ethanol/water droplet and an INN/ethanol precursor droplet both with 0.975 initial mass fractions of ethanol and an initial water or INN mass fraction of 0.025 , respectively. For the INN/ethanol droplet, the initial mass fraction of water in INN is 0.011 and that of IN is 0.014 . The total mass evaporation rate (left part of Fig. 4) shows an initial increase during the droplet heating period. During droplet heating, thermal expansion of the droplet occurs as seen in the right part of Fig. 4 where the normalized droplet surface area increases initially until it reaches its peak. After the quasi-steady droplet evaporation period, thermal decomposition occurs in the case of the INN/ethanol droplet whereas in the other two situations, the droplet completely evaporates. The initial evaporation is fastest and most pronounced for the pure ethanol droplet compared to the ethanol/water droplet with a mass fraction of 0.975 of ethanol and the INN/ethanol droplet. The preferential evaporation of ethanol is observed because ethanol is more volatile than water. Thus, the peak in the profile of the mass evaporation rate occurs earliest for the pure ethanol droplet. The heating of the INN/ethanol droplet is slower compared to the other droplets because of the different transport properties of the iron(III) nitrate and water. The entire evaporation process is fastest for the INN/ethanol droplet since after the complete evaporation of the ethanol and water, the iron(III) nitrate particle is left behind which is evident from the comparatively larger normalized droplet surface area. Also, the pure ethanol droplet evaporates faster than the ethanol/water and the INN/ethanol droplet.

Figure 5 shows the mass fraction and temperature plot at the droplet surface for the heating and evaporation of the INN/ethanol droplet in convective dry air. After the initial heating period, the quasi steady droplet evaporation occurs and beyond a temperature of $403 \mathrm{~K}$, thermal decomposition of the iron(III) nitrate occurs and transforms it into the gas phase as can be seen in the zoom in the right part of the figure. After the ethanol and water have completely evaporated at about $0.388 \mathrm{~ms}$, the latter step is the only one occurring in the process. During this last period, the temperature in the system is constant since the energy equation is determined by droplet evaporation.

The droplet evaporation time is about $0.39 \mathrm{~ms}$ and the time taken for the thermal decomposition of iron(III) nitrate into the gas phase in approximately $0.01 \mathrm{~ms}$. After evaporation, the maximum temperature, $T_{\mathrm{d}, \max }$, is reached and maintained
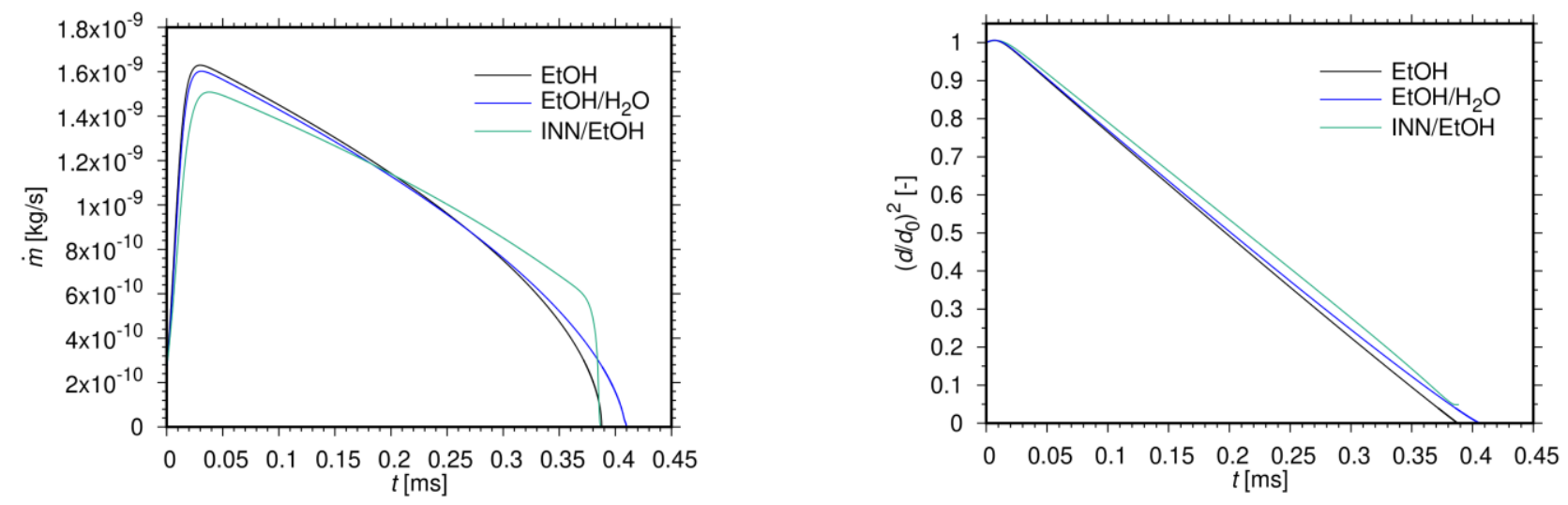

Fig. 4: Initial conditions: $r_{\mathrm{d}, 0}=5 \mu \mathrm{m}, T_{1,0}=293.15 \mathrm{~K}, p=1$ bar, $T_{\mathrm{g}, 0}=1200 \mathrm{~K}, u_{0}=4 \mathrm{~m} / \mathrm{s}, \mathrm{RH}=0 \%$. EtOH: $Y_{\mathrm{EtOH}, 0}=1$. EtOH/ $/ \mathrm{H}_{2} \mathrm{O}$ : $Y_{\mathrm{EtOH}, 0}=0.975, \mathrm{Y}_{\mathrm{H} 2 \mathrm{O}, 0}=0.025 . \mathrm{EtOH} / \mathrm{INN}: Y_{\mathrm{EtOH}, 0}=0.975, Y_{\mathrm{H} 2 \mathrm{O}, 0}=0.011, Y_{\mathrm{IN}, 0}=0.014$. Left: Mass evaporation rate with time. Right: Normalized droplet surface area with time. 

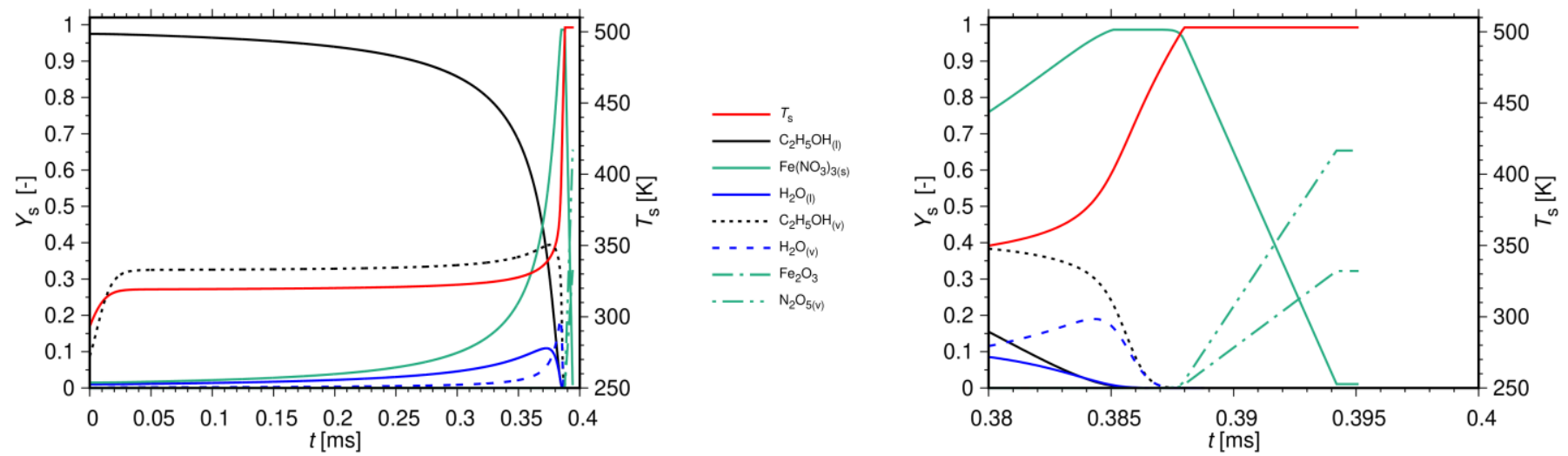

Fig. 5: Initial conditions: $r_{\mathrm{d}, 0}=5 \mu \mathrm{m}, T_{1,0}=293.15 \mathrm{~K}, p=1$ bar, $T_{\mathrm{g}, 0}=1200 \mathrm{~K}, u_{0}=4 \mathrm{~m} / \mathrm{s}, \mathrm{RH}=0 \%, Y_{\mathrm{EtOH}, 0}=0.975, Y_{\mathrm{H} 2 \mathrm{O}, 0}=0.011$, $Y_{I \mathrm{~N}, 0}=0.014$. Left: Mass fractions of species and gas temperature at the droplet surface. Right: Zoomed view of the mass fractions of species and of gas temperature at the droplet surface.
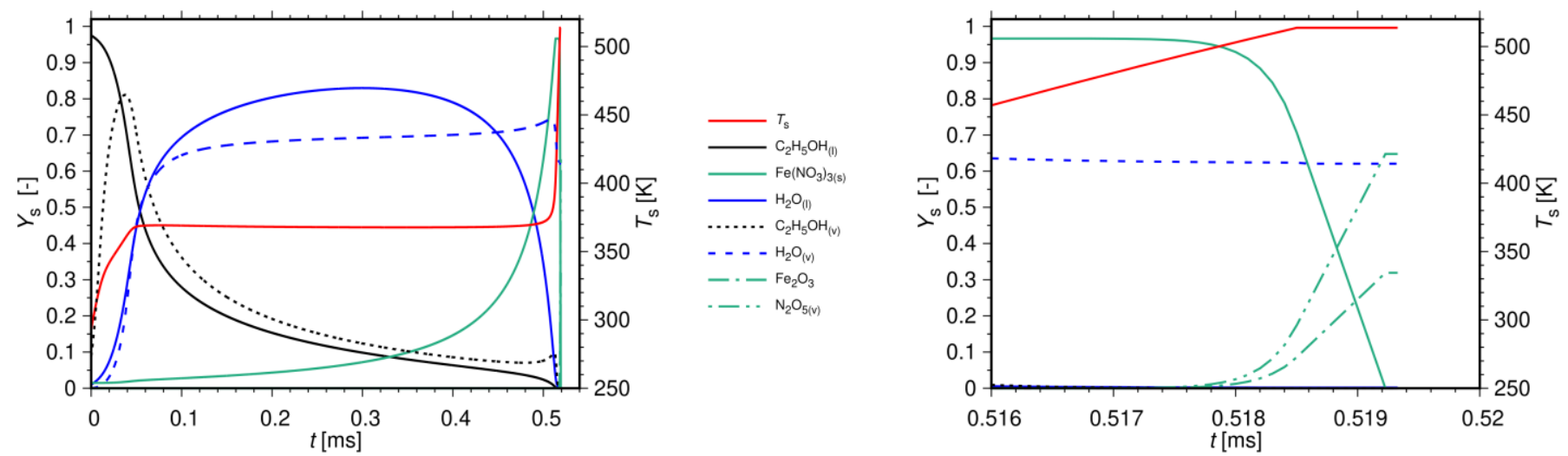

Fig. 6: Initial conditions: $r_{\mathrm{d}, 0}=5 \mu \mathrm{m}, T_{1,0}=293.15 \mathrm{~K}, p=1 \mathrm{bar}, T_{\mathrm{g}, 0}=1200 \mathrm{~K}, u_{0}=4 \mathrm{~m} / \mathrm{s}, \mathrm{RH}=10 \%, Y_{\mathrm{EtOH}, 0}=0.975, Y_{\mathrm{H} 2 \mathrm{O}, 0}=0.011$, $Y_{I \mathrm{~N}, 0}=0.014$. Left: Mass fractions of species and gas temperature at the droplet surface. Right: Zoomed view of the mass fractions of species and of gas temperature at the droplet surface.

throughout the entire thermal decomposition of iron(III) nitrate. The rate of thermal decomposition of iron(III) nitrate into gaseous components is exponentially with respect to the surface temperature, cf. Eq. (5). The higher the temperature during the droplet evaporation, the more enhanced is the thermal decomposition rate, leading to lower thermal decomposition times.

Figure 6 presents the mass fraction and temperature at the droplet surface of an INN/ethanol droplet with relative humidity of $10 \%$ in the ambient air. The relative humidity of $10 \%$ significantly affects the evaporation characteristics. The initially enhanced ethanol evaporation results in an increased water content at the droplet surface compared to dry air, see left part of Fig. 5. The droplet evaporation time is about $0.52 \mathrm{~ms}$, and the thermal decomposition time to transfer iron(III) nitrate into the gas phase in approximately $0.006 \mathrm{~ms}$.

Overall, the total process time to transfer the INN/ethanol precursor droplet completely into the gas phase is $0.4 \mathrm{~ms}$ in dry air versus about $0.53 \mathrm{~ms}$ in $10 \%$ humid air where the evaporation time is prolonged in humid air compared to dry air and the thermal decomposition time is reduced which is associated with the higher surface temperature of $513 \mathrm{~K}$ under humid conditions versus $502 \mathrm{~K}$ in dry air. 


\section{Summary and Conclusions}

A numerical study of the heating and evaporation and possible thermal decomposition of INN/ethanol precursor in humid and dry convective air has been performed for the formation of nanoparticles in spray flames. In low ambient temperatures below the thermal decomposition temperature of INN, the particles form directly inside the droplet whereas higher ambient temperature, thermal decomposition occurs, transferring the entire liquid into gaseous $\mathrm{Fe}_{2} \mathrm{O}_{3}$ and $\mathrm{N}_{2} \mathrm{O}_{5}$ which nanoparticle formation from the gas phase may occur which is typical for nanoparticle formation in spray flame pyrolysis. At both low and high ambient gas temperature, water condensation occurs at the droplet surface, leading to somewhat higher droplet surface temperatures but prolonging the evaporation process. For gas temperatures above the thermal decomposition temperature, the higher droplet surface temperature enhances thermal decomposition in humid compared to dry air.

\section{Acknowledgements}

The authors thank M. Nanjaiah, Prof. I. Wlokas, and Prof. H. Wiggers from Universität Essen-Duisburg for many helpful discussions concerning the liquid phase reactions. Funding by the Deutsche Forschungsgemeinschaft (DFG, German Research Foundation) - Projektnummer 374463455 and through HGS MathComp is gratefully acknowledged.

\section{References}

[1] C. S. Lindberg, M. Y. Manuputty, J. Akroyd, and M. Kraft, "A two-step simulation methodology for modelling stagnation flame synthesised aggregate nanoparticles," Combustion and Flame, vol. 202, pp. 143-153, 2019.

[2] R. Koirala, S. Pratsinis, and A. Baiker, "Synthesis of catalytic materials in flames: opportunities and challenges," Chem. Soc. Rev., vol. 45, pp. 3053-3068, 2016.

[3] P. B. Neto, L. Buss, F. Meierhofer, H. F. Meier, U. Fritsching, and D. Noriler, "Combustion kinetic analysis of flame spray pyrolysis process," Chemical Engineering and Processing-Process Intensification, vol. 129, pp. 17-27, 2018.

[4] W. Y. Teoh, R. Amal, and L. Mädler, "Flame spray pyrolysis: An enabling technology for nanoparticles design and fabrication," Nanoscale, vol. 2, pp. 1324-1347, 2010.

[5] A. Rittler, L. Deng, I. Wlokas, and A. Kempf, "Large eddy simulations of nanoparticle synthesis from flame spray pyrolysis," Proceedings of the Combustion Institute, vol. 36, 1077-1087, 2017.

[6] M. C. Heine and S. E. Pratsinis, "Droplet and particle dynamics during flame spray synthesis of nanoparticles," Industrial \& Engineering Chemistry Research, vol. 44, 6222-6232, 2005.

[7] B. Abramzon and W. Sirignano, "Droplet vaporization model for spray combustion calculations," International Journal of Heat and Mass Transfer, vol. 32, 1605-1618, 1989.

[8] G. Brenn, L. Deviprasath, F. Durst, and C. Fink, "Evaporation of acoustically levitated multi-component liquid droplets," International Journal of Heat and Mass Transfer, vol. 50, 5073-5086 , 2007.

[9] F. Schneider, S. Suleiman, J. Menser, E. Borukhovich, I. Wlokas, A. Kempf, H. Wiggers, and C. Schulz, "Spraysyn-a standardized burner configuration for nanoparticle synthesis in spray flames," Rev. Sci. Instruments 90, 085108, 2019.

[10] A. Gadalla and H. Yu, "Thermal decomposition of fe(iii) nitrate and its aerosol," J. Materials Res. 5, 1233-1236, 1990.

[11] J. Mu and D. Perlmutter, "Thermal decomposition of metal nitrates and their hydrates," Thermochimica Acta, pp. 253260, 1982.

[12] P. Narasu, S. Boschmann, P. Pöschko, F. Zhao, and E. Gutheil. "Modeling and simulation of single ethanol/water droplet evaporation in dry and humid air," Combustion Science and Technology 192, vol. 7, pp. 1233-1252, 2020.

[13] A. Keller, I. Wlokas, M. Kohns, and H. Hasse, "Thermophysical properties of solutions of iron(III)nitrate-nonahydrate in mixtures of ethanol and water," Journal of Chemical \& Engineering Data, vol. 65, pp. 3519-3527, 2020.

[14] B. E. Poling, J. M. Prausnitz, and J. P. O'Connell, The properties of gases and liquids. McGraw-Hill New York, 2001.

[15] M. Kleiber and R. Joh, "Berechnungsmethoden für thermophysikalische Stoffeigenschaften.," in: P. Stephan, D. Mewes, S. Kabelac, M. Kind, K. Schaber, and T. Wetzel, (Eds.), VDI-Wärmeatlas. Springer Vieweg, Heidelberg 2018, 1-45.

[16] J. Gmehling, P. Rasmussen, and A. Fredenslund, "Vapor-liquid equilibriums by UNIFAC group contribution. Revision and Extension 2," Industrial \& Engineering Chemistry Process Design and Development, vol. 21, pp. 118-127, 1982. 